\title{
Continuous exposure of non-small cell lung cancer cells with wild-type EGFR to an inhibitor of EGFR tyrosine kinase induces chemoresistance by activating STAT3
}

\author{
JIE TANG ${ }^{1}$, FUCHUN GUO $^{2}$, YANG DU ${ }^{1}$, XIAOLING LIU ${ }^{2}$, QING QIN $^{2}$, \\ XIAOKE LIU $^{2}$, TAO YIN ${ }^{3}$, LI JIANG ${ }^{1}$ and YONGSHENG WANG ${ }^{2}$
}

Departments of ${ }^{1}$ Abdominal Oncology and ${ }^{2}$ Thoracic Oncology, Cancer Center and State Key Laboratory of Biotherapy, West China Hospital; ${ }^{3}$ State Key Laboratory of Biotherapy, Sichuan University, Chengdu, Sichuan 610041, P.R. China

Received October 16, 2014; Accepted December 10, 2014

DOI: $10.3892 / \mathrm{ijo} .2015 .2898$

\begin{abstract}
Epidermal growth factor receptor-tyrosine kinase inhibitors (EGFR-TKIs) have shown promising effects against the growth of non-small cell lung cancer (NSCLC) cells harboring EGFR mutations (EGFR-mts). However, many patients with NSCLC that are accepted for EGFR-TKI treatment followed by chemotherapy possess an unknown EGFR status including wild-type EGFR (EGFR-wt). Little is known about the potential effects of EGFR-TKI treatment prior to chemotherapy. We investigated the effects and underlying molecular events of 4 weeks of continuous exposure to EGFR-TKIs in the EGFR-wt NSCLC line H1299. This treatment dramatically increased the $\mathrm{IC}_{50}$ of several relevant chemotherapeutic agents: cisplatin (DDP) $(29.25 \pm 6.1 \mu \mathrm{M}$ for gefitinib, $43.25 \pm 14.87 \mu \mathrm{M}$ for erlotinib, and $6.92 \pm 1.15 \mu \mathrm{M}$ for parental), paclitaxel (11.16 $\pm 3.36 \mu \mathrm{M}$ for gefitinib, $9.16 \pm 1.41 \mu \mathrm{M}$ for erlotinib, and $2.09 \pm 0.44 \mu \mathrm{M}$ for parental), gemcitabine (47.18 $\pm 6.2 \mu \mathrm{M}$ for gefitinib, $40.36 \pm 11.1 \mu \mathrm{M}$ for erlotinib, and $16.00 \pm 3.38 \mu \mathrm{M}$ for parental) and pemetrexed $(11.78 \pm 4.07 \mu \mathrm{M}$ for gefitinib, $15.97 \pm 7.23 \mu \mathrm{M}$ for erlotinib, and $4.72 \pm 1.9 \mu \mathrm{M}$ for parental). This chemoresistance was critically dependent on the activation of the mediator signal transducer and activator of transcription 3 (STAT3). In cells exposed to EGFR-TKIs for 4 weeks, activation of STAT3 was found to be unrelated to EGFR and to be independent of IL- 6 and -22 . Treatment with the STAT3 inhibitor NSC 74859 was able to reverse the TKI exposure-induced chemoresistance in EGFR-wt NSCLC cells. Similar phenomena were observed in H1975 cells harboring
\end{abstract}

Correspondence to: Dr Yongsheng Wang, Department of Thoracic Oncology, Cancer Center and State Key Laboratory of Biotherapy, West China Hospital, Sichuan University, No. 37 Guo Xue Xiang, Chengdu, Sichuan 610041, P.R. China

E-mail:cc300j@gmail.com

Key words: non-small cell lung cancer, epidermal growth factor receptor, gefitinib, erlotinib, signal transducer and activator of transcription 3 , chemoresistance
EGFR L858R and T790M mutations. Based on the observed molecular events following long exposure of an EGFR-wt NSCLC cell line to an EGFR-TKI, this study indicates that such drugs should be not recommended for EGFR-wt patients who can undergo chemotherapy. This study also suggests that STAT3 inhibitors may aid in the treatment NSCLC patients who exhibit EGFR-TKI resistance due to an acquired T790M mutation.

\section{Introduction}

Non-small cell lung cancers (NSCLCs) with activating mutations in the tyrosine kinase domain (TKD) of EGFR have been reported to exhibit 'oncogene addiction' to reflect their dependence on EGFR-mediated malignant biological behavior $(1,2)$. Several clinical trials have shown that epidermal growth factor receptor-tyrosine kinase inhibitors (EGFR-TKIs) (e.g., gefitinib and erlotinib) are the best front-line options for patients with sensitive EGFR mutations (EGFR-mts), resulting in a 2-3-fold prolongation of survival time compared with standard chemotherapy (3-5). For patients with wild-type EGFR (EGFR-wt) status, data from randomized trials suggested that some of these patients will derive a modest benefit from these agents.

Currently, first-line use of these agents should be restricted to EGFR-mt-positive patients as a clinical practice guideline in the treatment of NSCLC (6). In practice, however, some EGFR status-unknown patients might also benefit from empirical use of initial treatment with EGFR-TKIs.

Most patients with NSCLC are diagnosed at stages III and IV (7). For those with advanced lung cancer that cannot be removed surgically, chemotherapy or molecular-targeting treatments are typically recommended.

It has been reported that EGFR-mts creating sensitivity to EGFR-TKIs are more common in Asian populations, particularly in patients with lung adenocarcinoma (8). Fine-needle aspirates for diagnosis, which are now commonly used, are often insufficient for molecular analysis. Accordingly, a number of technical issues may confound the analysis of EGFR-mts. In addition, $>60 \%$ of NSCLCs show overexpression of EGFR (9), and numerous investigations have shown that 
EGFR-TKIs can inhibit TKD activation of EGFR-wt in vitro. Moreover, many NSCLC patients are more inclined to undergo EGFR-TKI treatment because they fear chemotherapy toxicity, especially patients with poor Eastern Cooperative Oncology Group (ECOG) performance status. In view of this, some oncologists usually offer EGFR-TKIs as a tentative treatment lasting for $\sim 1$ month in these patients.

Although details of subsequent treatments and response rates for chemotherapy (as the second-line treatment) following first-line EGFR-TKI treatment in patients with EGFR-wt NSCLC are not available from the IPASS and First-SIGNAL trials, the overall survival (OS) advantage of patients with standard first-line chemotherapy indirectly suggests that prior treatment with EGFR-TKIs might result in unwanted effects (8). The TORCH study, a phase III trial performed in unselected NSCLC patients, most of whom were EGFR-wt, addressed a sequence question by using a crossover design that compared first-line erlotinib followed by cisplatin (DDP)-gemcitabine at progression and comparing this with the reverse strategy (10). This study found that starting with erlotinib not only decreased the objective response rate (ORR), but also led to worse survival in EGFR-wt NSCLC patients (mOS: 6.5 vs. 9.6 months). Moreover, a retrospective study to investigate the prognosis of patients with NSCLC receiving second-line antitumor treatment after gefitinib therapy showed that no survival benefits from platinum-based combination regimens existed in patients with EGFR-wt NSCLC (11). These findings led us to investigate whether initial EGFR-TKI treatment has an adverse effect on the sensitivity to subsequent chemotherapy of EGFR-wt NSCLC, and to explore the underlying mechanisms.

The tentative treatment may increase the risk of patients with EGFR-wt having an unfavorable prognosis, including a significantly reduced total progression-free survival (PFS) and OS. Here, we describe the first study focusing on the effectiveness of chemotherapy following continuous exposure of EGFR-wt NSCLC to EGFR-TKIs in vitro.

\section{Materials and methods}

Reagents. RPMI-1640 medium, fetal bovine serum, trypsin, penicillin and streptomycin were obtained from Gibco/Life Technologies (Shanghai, China). Gefitinib(Iressa) was provided by AstraZeneca (London, UK), erlotinib (Tarceva) was a gift from Roche Pharmaceuticals (Basel, Switzerland), pemetrexed (Alimta) and gemcitabine (Gemzar) were a gift from Eli Lilly and Company (Indianapolis, IN, USA). DDP and paclitaxel (Taxol) were purchased from Sigma-Aldrich (St. Louis, MO, USA). The drugs were dissolved in dimethyl sulfoxide (DMSO) or sterile water and diluted in culture medium before use. NSC 74859, an inhibitor of signal transducer and activator of transcription 3 (STAT3) was purchased from Selleck Chemicals (Houston, TX, USA). LY294002, AS605240 and leptomycin B an inhibitor of nuclear export were purchased from Sigma-Aldrich. Recombinant human EGF was purchased from BioLegend (San Diego, CA, USA).

Cell culture and long-term exposure to TKI. The NSCLC cell lines, HCC827 [lung adenocarcinoma with an acquired mutation in the EGFR TKD (E746-A750 deletion)], NCI-H1299 (established from a lymph node metastasis of the lung from a patient who had received prior radiation therapy and with EGFR-wt) and NCI-H1975 (primary adenocarcinoma harboring EGFR L858R and T790M mutations) were purchased from the American Type Culture Collection (ATCC) (Manassas, VA, USA) and maintained in RPMI-1640 medium supplemented with $10 \%$ FBS and antibiotics. We also established a model of EGFR-TKIs exposure of lung cancer by culturing $\mathrm{H} 1299$ in $10 \mu \mathrm{M}$ gefitinib and $5 \mu \mathrm{M}$ erlotinib respectively for 4 weeks as well as H1975; HCC827 was incubated in $2 \mu \mathrm{M}$ gefitinib for 6 months.

Growth inhibition assay. The number of viable cells was estimated using the Cell Counting kit-8 (Dojindo, Kumamoto, Japan) assay that provided effective and reproducible determination of the proliferative activity of cells. Human cells were seeded into flat-bottomed 96 -well microplates at a density of $10^{4}$ cells/well in $100 \mu \mathrm{l}$ of culture medium and allowed to attach to the wells overnight before $100 \mu \mathrm{l}$ medium containing $2 \mathrm{x}$ indicated concentration of EGFR-TKIs, with or without a STAT3 inhibitor, was added to each well. After $24 \mathrm{~h}$, the media were separately replaced with fresh medium containing each cytotoxic drug (pemetrexed, gemcitabine, DDP, paclitaxel) which dissolved at variously gradient concentrations. Cells were treated with chemotherapeutic drugs for $48 \mathrm{~h}$. Controls without cytotoxic drug exposure were included in each experiment. Five replicate wells were used for each drug concentration and each experiment was carried out independently three times. To measure the proliferative activity of cells in 96-well microplates, CCK-8 reagent was added ( $20 \mu \mathrm{l} /$ well) and incubation continued for $2 \mathrm{~h}$. Absorbance of the reduced formazan was measured at $450 \mathrm{~nm}$ using a microplate reader (Multiskan MK3; Thermo Fisher Scientific, Inc., Waltham, MA, USA) with a reference wavelength of $650 \mathrm{~nm}$.

Caspase-3 activity assay. Caspase-3 activity was determined after treatment of cells with TKI and cytotoxic drugs as described for the growth inhibition assay. Cell lysates were prepared by the PathScan Sandwich ELISA Lysis buffer and the activity of caspase-3 was determined using a Caspase-3 Activity Assay kit (both from Cell Signaling Technology, Inc., Danvers, MA, USA) that assesses cleavage of the fluorogenic peptide. After treatment with cytotoxic drugs, cells (including those loosely attached to the plate) were collected and rinsed with ice-cold PBS. The fluorescence of cleaved AMC was assessed after $1 \mathrm{~h}$ at $37^{\circ} \mathrm{C}$ incubation in the dark. Caspase-3 activity values were calculated from a standard curve generated by using varying concentrations of AMC (Microsoft Excel; Microsoft, Redmond, WA, USA).

FACS analysis and apoptosis assay. Cells were fixed in ice-cold $70 \%$ ethanol and stained with propidium iodide (50 mg/ml in PBS; Sigma-Aldrich) in the presence of RNase A $(100 \mathrm{mg} / \mathrm{ml})$ for DNA content analysis by flow cytometry using a FACSCalibur system (BD Biosciences, San Diego, CA, USA). For each data point, 8,000 cells were analyzed. The percentage of cells in various phases of the cell cycle was calculated using FlowJo software version 7.6.1 (Tree Star, Inc., Ashland, OR, USA). Apoptosis was quantified 
using an Annexin V-FITC Apoptosis kit (BD Biosciences) in accordance with the manufacturer's instructions. In brief, cells were trypsinized, pelleted by centrifugation $(1,500 \mathrm{rpm}$ for 5 min, Heraeus Multifuge X3; Thermo Fisher Scientific, Inc.) and resuspended in Annexin V-binding buffer. FITC-conjugated Annexin V and propidium iodide were added to cells and incubated for $30 \mathrm{~min}$ at room temperature in the dark. Analyses were done on a FACSCalibur system (BD Biosciences) and FlowJo software version 7.6.1 (Tree Star, Inc.)

Preparation of nuclear and cytoplasmic protein extracts. Nuclear extracts from cells were isolated using a Nuclear and Cytoplasmic Protein Extraction kit in accordance with the manufacturer's instructions (Beyotime Institute of Biotechnology, Jiangsu, China). In brief, cells were washed in ice-cold PBS then collected and resuspended by pipetting up and down 10 times in $200 \mu \mathrm{l}$ of ice-cold cell lysis buffer. After resting on ice for $15 \mathrm{~min}$, nuclei were pelleted in a microcentrifuge (Sorvall Legend Micro; Thermo Fisher Scientific, Inc.) at $12,000 \mathrm{rpm}$ for $5 \mathrm{~min}$ at $4^{\circ} \mathrm{C}$ and the cytoplasmic supernatants were aliquoted and stored at $-80^{\circ} \mathrm{C}$ for western blot analysis when needed. Pelleted nuclei were then resuspended in $50 \mu \mathrm{l}$ of nuclear extraction buffer. After intermittently vortexing (vortex $30 \mathrm{sec}$, rest $30 \mathrm{sec}$ ) the mixing for $30 \mathrm{~min}$ and centrifugation at $12,000 \mathrm{rpm}$ for $10 \mathrm{~min}$ at $4^{\circ} \mathrm{C}$, nuclear extracts were aliquoted and stored at $-80^{\circ} \mathrm{C}$ until use. The concentration of proteins in the cytoplasmic and nuclear extracts were measured using a BCA Protein Assay kit (Beyotime Institute of Biotechnology).

Western blot analyses. Cells were lysed using the PhosphoSafe Extraction Reagent (Novagen; EMD Biosciences, San Diego, CA, USA) supplemented with a cocktail of protease inhibitors and $1 \mathrm{mM}$ phenylmethylsulfonyl fluoride (Sigma-Aldrich). Protein extracts were heated in protein loading buffer (Beyotime Institute of Biotechnology) at $95^{\circ} \mathrm{C}$ for $5 \mathrm{~min}$ and separated by SDS-PAGE. After electrophoresis, separated proteins were electrotransblotted onto a PVDF membrane and then blocked using 1\% BSA in TBS-Tween-20 for $2 \mathrm{~h}$ at room temperature. The membrane was incubated overnight at $4^{\circ} \mathrm{C}$ with a primary antibody prior to use of a horseradish peroxidase (HRP)-labeled secondary antibody, and visualization of bands by chemiluminescence, recorded with X-OMAT BT film [Kodak (China) Co., Ltd., Fujian, China]. Details of the primary antibodies used are given in Table I.

Xenograft model. Female nude mice with a BALB/c genetic background were purchased from HuaFukang Biological Technology Co., Ltd. (Beijing, China). Mice aged 4-6 weeks, 18-22 $\mathrm{g}$ in weight, were maintained under specific pathogen-free conditions with 12-h light/12-h dark cycles at $26-28^{\circ} \mathrm{C}$ and $50-65 \%$ humidity in the Experimental Animal Centre of the Sichuan University State Key Laboratory of Biotherapy (Sichuan, China) for these experiments. Each five animals were housed in plastic containers with lids. All animals were checked daily; containers were changed once a week during the entire length of the experiment. Animal feed and underpad, which were purchased from the HuaFukang Biological Technology Co., Ltd., were autoclaved and vacuum packed. The water was sterilized and then adjusted to room temperature before use. H1299 cells were used for the xenograft experiment. In brief, H1299 cells $\left(1 \times 10^{7}\right.$ cells/each mouse) were implanted subcutaneously in the right axilla of nude mice. Drug treatments were started on day 28. Gefitinib $(100 \mathrm{mg} / \mathrm{kg})$ or erlotinib $(100 \mathrm{mg} / \mathrm{kg})$ was given by oral gavage 5 times/week. In total three treatment cycles were conducted. Each treatment group contained 10 mice. Mice were euthanized by cervical dislocation, and tumor tissues were rapidly dissected; part of them flash-frozen in liquid nitrogen, for later protein extraction, the others formalin-fixed $24 \mathrm{~h}$ and then paraffin-embedded. All procedures were approved by the Animal Care and Use Committee of Sichuan University.

Immunohistochemical staining. The formalin-fixed paraffin-embedded tissue samples of the tumor were cut into sections of $4 \mu \mathrm{m}$, which were mounted on silanized slides. The sections were deparaffinized in xylene then rehydrated through a graded series of ethanol/water. Antigen retrieval was accomplished using $\mathrm{pH} 6.0$ sodium citrate buffer $(0.01 \mathrm{M})$ and microwave heating for $10 \mathrm{~min}$ at $95^{\circ} \mathrm{C}$. After cooling, the sections were incubated with a primary antibody at $4^{\circ} \mathrm{C}$ overnight (Table I). The PowerVision 6000 immunohistochemistry detection reagent (ZSJQ Biotechnology, Beijing, China) was used as a second antibody by incubating for $1 \mathrm{~h}$ at $37^{\circ} \mathrm{C}$ and 3,3'-diaminobenzidine (DAB) was used as a chromogen. Hematoxylin was used as a nuclear counterstaining agent.

Cytokine assays. EGFR-TKI-exposed or parental cells were plated in their respective growth media at $1 \times 10^{5}$ cells/well and incubated overnight for attachment. The media were replaced with fresh serum-free medium for serum-starved and EGFR-TKI-exposed (parental cells without EGFR-TKIs).

After $48 \mathrm{~h}$ of EGFR-TKI exposure, conditioned medium was then harvested and stored at $-80^{\circ} \mathrm{C}$. Culture medium incubated without cells served as the control. The conditioned medium was thawed and centrifuged briefly before assay.

Quantification of IL-6 and -22 in cell culture supernatants was carried out using an ELISA Development kit (Quantikine Colorimetric Sandwich ELISAs; R\&D Systems, Minneapolis, MN, USA) in microplate format, measuring absorbance at $450 \mathrm{~nm}$ and with wavelength correction at $570 \mathrm{~nm}$ for correct optical imperfections in the plates.

Immunoprecipitation. The physical interaction between STAT3 and EGFR was detected by immunoprecipitation. Cells were lysed in non-denaturing lysis buffer containing $20 \mathrm{mM}$ Tris (pH 7.5), $150 \mathrm{mM} \mathrm{NaCl}, 1 \%$ Triton X-100, supplemented with a protease and phosphatase inhibitor cocktail (nos. P8340 and P0044; Sigma-Aldrich). Samples were precleared with rabbit IgG (Santa Cruz Biotechnology, Inc., Santa Cruz, CA, USA). Each sample supernatant was then incubated with antibodies at a dilution ratio indicated in the instructions at $4{ }^{\circ} \mathrm{C}$ overnight with gentle agitation. The samples were further incubated with $40 \mu \mathrm{l}$ of Protein A/G PLUS Agarose beads (Santa Cruz Biotechnology, Inc.) for $4 \mathrm{~h}$ at $4^{\circ} \mathrm{C}$ and the resulting immune complexes were washed three times with lysis buffer by centrifugation (800 rpm, $3 \mathrm{~min}$ ). Samples were heated in SDS loading buffer at $95^{\circ} \mathrm{C}$ for $5 \mathrm{~min}$ and analyzed by western blot analysis. 
Table I. Details of the primary antibodies.

\begin{tabular}{|c|c|c|c|c|}
\hline Primary antibody & Clone & Dilution (WB) & Catalog & Supplier \\
\hline FGFR2 & Polyclonal rabbit & $1: 2,000$ & ab10648 & Abcam \\
\hline IGF-1R & Monoclonal rabbit & $1: 2,000$ & ab172965 & Abcam \\
\hline IGFBP3 & Polyclonal rabbit & $1: 2,000$ & ab76001 & Abcam \\
\hline IRS-1 & Polyclonal rabbit & $1: 2,000$ & ab52167 & Abcam \\
\hline mTOR & Polyclonal rabbit & $1: 2,000$ & $\mathrm{ab} 2732$ & Abcam \\
\hline mTOR (phospho S2448) & Polyclonal rabbit & $1: 2,000$ & ab84400 & Abcam \\
\hline P-STAT3 (Ser727) & Monoclonal rabbit & $1: 2,000$ & ab32143 & Abcam \\
\hline Bcl-2 & Monoclonal rabbit & $1: 1,000$ & no. 2870 & CST \\
\hline Bcl-xL & Monoclonal rabbit & $1: 1,000$ & no. 2764 & CST \\
\hline Cleaved PARP & Monoclonal rabbit & $1: 1,000$ & no. 5625 & CST \\
\hline c-MET & Polyclonal rabbit & $1: 1,000$ & no. 4560 & CST \\
\hline c-Myc & Monoclonal rabbit & $1: 1,000$ & no. 5605 & CST \\
\hline Cyclin D1 & Monoclonal rabbit & $1: 1,000$ & no. 2978 & CST \\
\hline E-cadherin & Monoclonal rabbit & $1: 1,000$ & no. 3195 & CST \\
\hline EGFR & Monoclonal rabbit & $1: 1,000$ & no. 4267 & CST \\
\hline GAPDH & Monoclonal rabbit & $1: 1,000$ & no. 5174 & CST \\
\hline HER-2 & Monoclonal rabbit & $1: 1,000$ & no. 4290 & CST \\
\hline Histone H3 & Monoclonal rabbit & $1: 1,000$ & no. 4499 & CST \\
\hline Mcl-1 & Monoclonal rabbit & $1: 1,000$ & no. 5453 & CST \\
\hline NF-кB p65 & Monoclonal rabbit & $1: 1,000$ & no. 4764 & CST \\
\hline P27Kip1 & Monoclonal rabbit & $1: 1,000$ & no. 3686 & CST \\
\hline P-AKT (Ser473) & Monoclonal rabbit & $1: 1,000$ & no. 4060 & CST \\
\hline P-Erk (Thr202/Tyr204) & Monoclonal rabbit & $1: 1,000$ & no. 4370 & CST \\
\hline P-IGF-1R $\beta$ (Tyr1131) & Polyclonal rabbit & $1: 1,000$ & no. 3021 & CST \\
\hline P-IGF-1R $\beta$ (Tyr1316) & Polyclonal rabbit & $1: 1,000$ & no. 6113 & CST \\
\hline P-JNK (Thr183/Tyr185) & Polyclonal rabbit & $1: 1,000$ & no. 4668 & CST \\
\hline P-MET (Tyr1234/1235) & Monoclonal rabbit & $1: 1,000$ & no. 3077 & CST \\
\hline P-NF-кB p65 (Ser536) & Monoclonal rabbit & $1: 1,000$ & no. 3033 & CST \\
\hline P-p38 (Thr180/Tyr182) & Polyclonal rabbit & $1: 1,000$ & no. 9211 & CST \\
\hline P-SFK (Tyr416) & Monoclonal rabbit & $1: 1,000$ & no. 6943 & CST \\
\hline P-STAT1 (Tyr701) & Monoclonal rabbit & $1: 1,000$ & no. 6943 & CST \\
\hline P-STAT1 (Tyr727) & Polyclonal rabbit & $1: 1,000$ & no. 9177 & CST \\
\hline P-STAT3 (Tyr705) & Monoclonal rabbit & $\begin{array}{c}1: 1,000 \\
\text { IHC } 1: 200\end{array}$ & no. 9145 & CST \\
\hline P- $\beta$-catenin $($ Ser 552$)$ & Polyclonal rabbit & $1: 1,000$ & no. 9566 & CST \\
\hline Snail & Monoclonal rabbit & $1: 1,000$ & no. 3879 & CST \\
\hline Total AKT & Polyclonal rabbit & $1: 1,000$ & no. 9272 & $\mathrm{CST}$ \\
\hline Total Erk & Monoclonal rabbit & $1: 1,000$ & no. 4695 & CST \\
\hline Total JNK & Monoclonal rabbit & $1: 1,000$ & no. 9258 & CST \\
\hline Total p38 & Polyclonal rabbit & $1: 1,000$ & no. 9212 & CST \\
\hline Total STAT1 & Polyclonal rabbit & $1: 1,000$ & no. 9172 & CST \\
\hline Total STAT3 & Monoclonal rabbit & $1: 1,000$ & no. 4904 & CST \\
\hline Vimentin & Monoclonal rabbit & $1: 1,000$ & no. 5741 & CST \\
\hline$\beta$-catenin & Monoclonal rabbit & $1: 1,000$ & no. 9582 & CST \\
\hline$\beta$-tubulin & Monoclonal rabbit & $1: 1,000$ & no. 2128 & CST \\
\hline Survivin & Monoclonal mouse & $1: 250$ & sc-17779 & $\mathrm{SCB}$ \\
\hline
\end{tabular}

STAT3, signal transducer and activator of transcription 3; PARP, poly(ADP-ribose) polymerase; CST, Cell Signaling Technology, Inc.; SCB, Santa Cruz Biotechnology, Inc. 
Table II. $\mathrm{IC}_{50}$ of $\mathrm{H} 1299$ for four cytotoxic drugs $(\mu \mathrm{M})$.

\begin{tabular}{|c|c|c|c|c|c|}
\hline & $\mathrm{P}$ & $\mathrm{G}$ & $\mathrm{E}$ & $\mathrm{G}+\mathrm{N}$ & $\mathrm{E}+\mathrm{N}$ \\
\hline DDP & $6.92 \pm 1.15$ & $29.25 \pm 6.1$ & $43.25 \pm 14.87$ & $14.12 \pm 3.13$ & $7.95 \pm 1.85$ \\
\hline Taxol & $2.09 \pm 0.44$ & $11.16 \pm 3.36$ & $9.16 \pm 1.41$ & $2.99 \pm 0.84$ & $3.16 \pm 0.91$ \\
\hline Gemzar & $16.00 \pm 3.38$ & $47.18 \pm 6.2$ & $40.36 \pm 11.1$ & $15.37 \pm 4.2$ & $23.23 \pm 2.3$ \\
\hline Alimta & $4.72 \pm 1.9$ & $11.78 \pm 4.07$ & $15.97 \pm 7.23$ & $9.3 \pm 1.7$ & $5.22 \pm 1.28$ \\
\hline
\end{tabular}

Data are shown as mean \pm SD. Experiments were repeated three times. P, parental; G, gefitinib-exposed; E, erlotinib-exposed; G+N, gefitinib-exposed + 24 h NSC 74859; E+N, erlotinib-exposed + 24 h NSC 74859; DDP, cisplatin; Taxol, paclitaxel; Gemzar, gemcitabine; Alimta, pemetrexed.
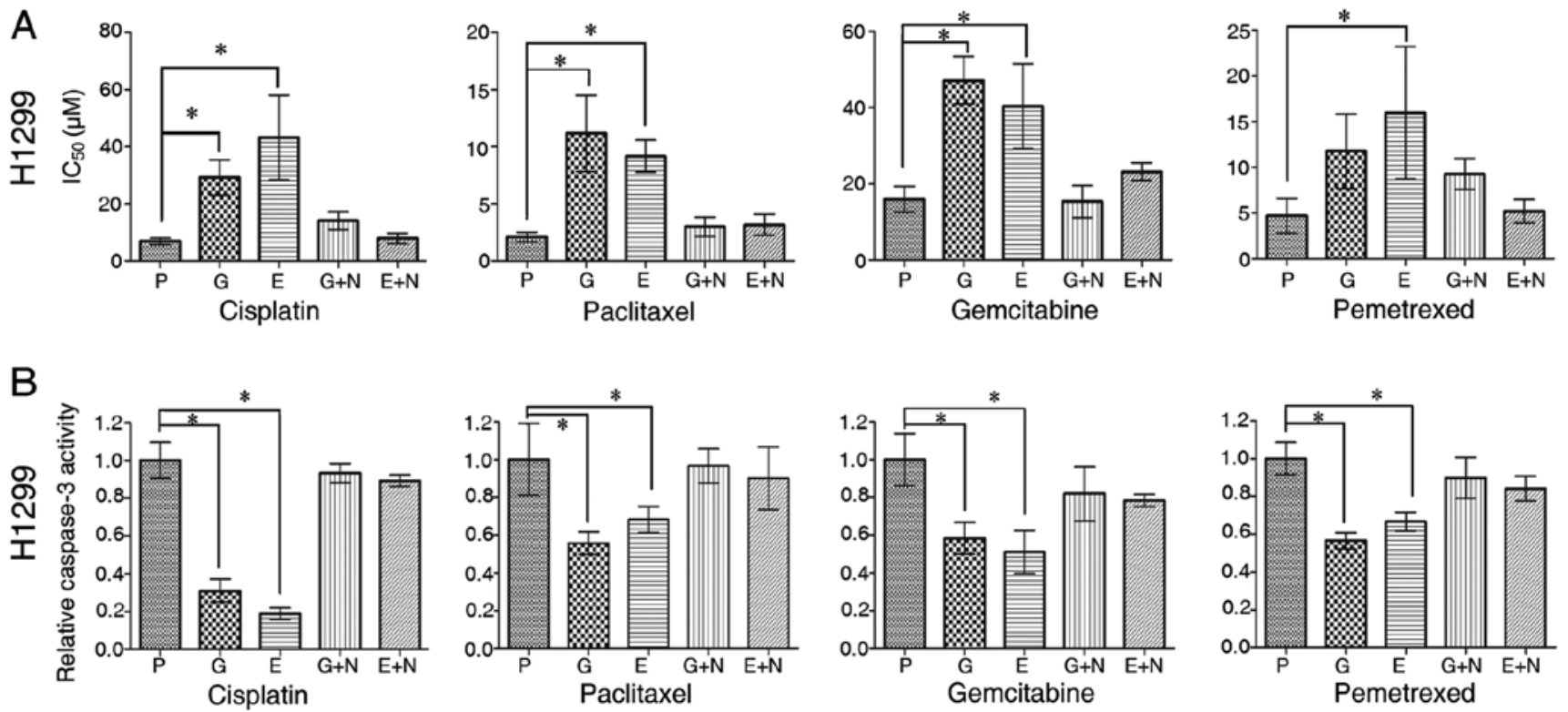

Figure 1. TKI-exposed-negative effect on cytotoxic drugs in wild-type EGFR (EGFR-wt) non-small cell lung cancer (NSCLC) cells. (A) The assessment of $\mathrm{IC}_{50}$ [cisplatin (DDP), paclitaxel, gemcitabine and pemetrexed] using the CCK-8 assay on parental and TKI (gefitinib and erlotinib)-exposed H1299 cells in the absence or presence of $20 \mu \mathrm{M} \mathrm{NSC} 74859$ for $24 \mathrm{~h}$. (B) H1299 NSCLC cells (parental, TKI-exposure and addition of NSC $7485920 \mu \mathrm{M}$ for $24 \mathrm{~h}$ ) were treated with DDP $(10 \mu \mathrm{M})$, paclitaxel $(1 \mu \mathrm{M})$, gemcitabine $(40 \mu \mathrm{M})$ and pemetrexed $(20 \mu \mathrm{M})$ for $24 \mathrm{~h}$ and subjected to caspase-3 assay. P, parental; G, gefitinib-exposed; E, erlotinib-exposed; G+N, Gefitinib-exposed $+24 \mathrm{~h}$ NSC 74859; E+N, Erlotinib-exposed $+24 \mathrm{~h}$ NSC 74859. Data are shown as mean \pm SD;

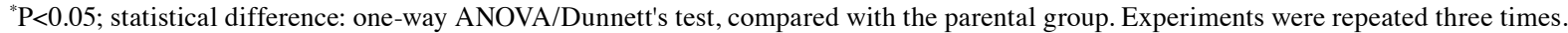

\section{Results}

Continuous exposure to EGFR-TKIs induces chemoresistance to cytotoxic agents in EGFR-wt NSCLC cell lines. To investigate the effects of EGFR-TKI exposure on chemotherapy, EGFR-wt NSCLC H1299 cells were continuously treated with gefitinib or erlotinib for 4 weeks. Chemosensitivity to DDP, paclitaxel, gemcitabine or pemetrexed in these NSCLC cells, both parental and TKI-exposed was evaluated. For each cytotoxic drug, the $\mathrm{IC}_{50}$ values obtained from TKI-exposed cells were significantly higher than from the parental cells (Fig. 1A, Table II). The difference was especially marked for gemcitabine after continuous exposure to TKI for 4 weeks.

To further assess the chemoresistance induced by TKI exposure, we assessed apoptosis induced by cytotoxic drugs. The proportion of apoptotic cells (including early and late phase) labeled with Annexin $\mathrm{V}(+)$ was decreased in all three TKI-exposed cell lines compared with their parental lines (Fig. 2A).
We also measured the caspase-3 activity of H1299 cells, and detected the expression of cleaved poly(ADP-ribose) polymerase (PARP) by western blot analysis. High levels of active caspase- 3 and proteolytic cleavage of PARP are two characteristic biochemical markers of apoptosis. The level of active caspase- 3 induced by each of the four cytotoxic drugs was attenuated after TKI exposure compared with the parental group (Fig. 1B). The cells pre-exposed to TKI for 4 weeks showed a reduced level of cleaved PARP when treated with cytotoxic drugs compared with their parental cells (Fig. 2B).

TKI exposure induces high-level activation of STAT3 in EGFR-wt NSCLC cells. To gain insights into the mechanisms underlying the resistance of cytotoxic agents after TKI exposure of EGFR-wt NSCLC cells, proteins of the EGFR signaling pathway were detected by western blot analysis. Given that EGFR signaling activation stimulates intracellular cascades, including the MAPK, PI3K/AKT, and STAT signaling pathways $(12,13)$, we analyzed the activity of several major EGFR 
A $\mathrm{H} 1299$
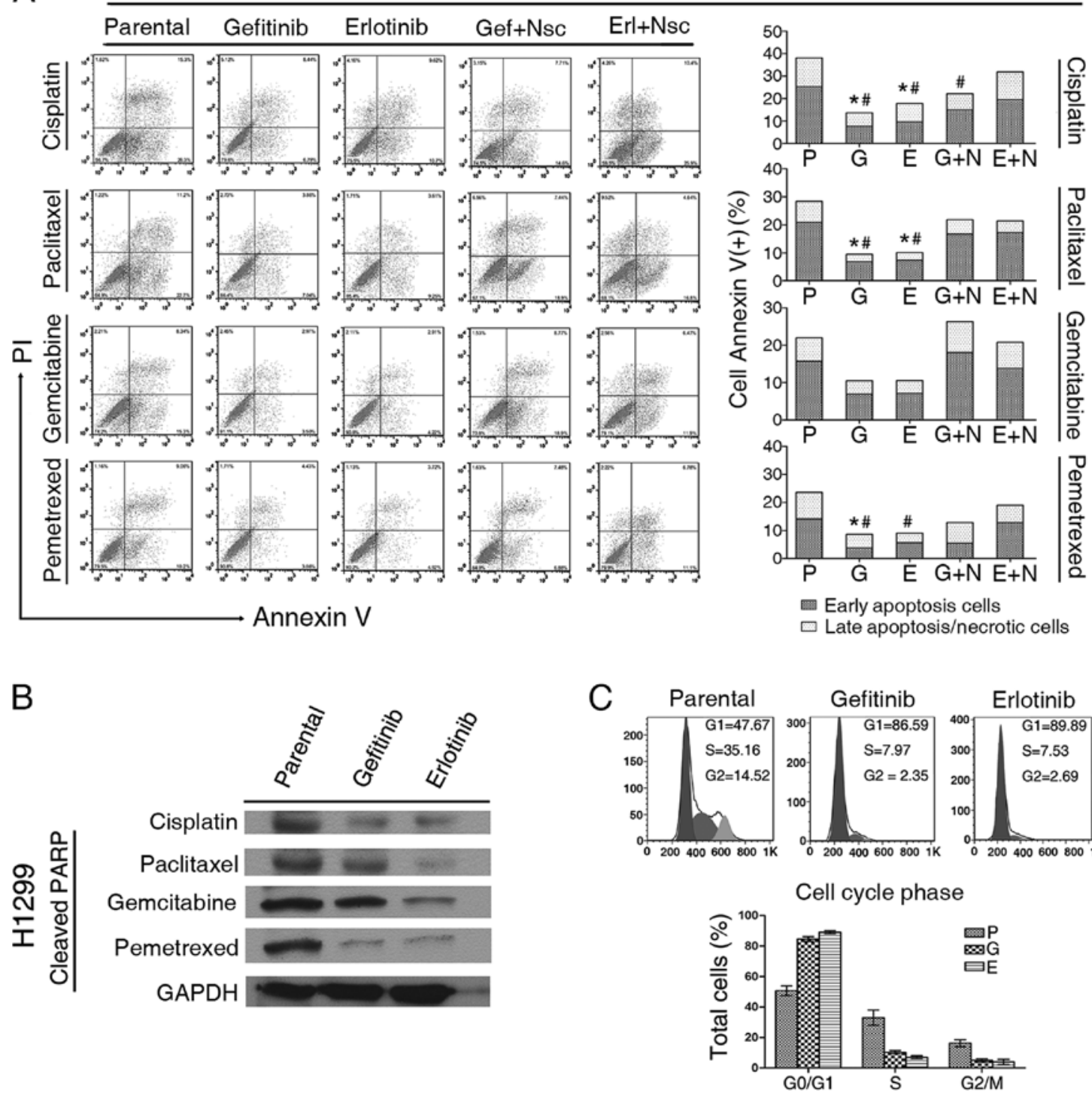

Figure 2. Analysis of apoptosis and cell cycle phase in cultured non-small cell lung cancer (NSCLC). (A) The apoptotic incidence for four chemotherapeutic agents [10 $\mu \mathrm{M}$ cisplatin (DDP), $1 \mu \mathrm{M}$ paclitaxel, $10 \mu \mathrm{M}$ gemcitabine or $5 \mu \mathrm{M}$ pemetrexed] after $48 \mathrm{~h}$ exposure of $\mathrm{H} 1299$ cells in five pre-treatment groups as indicated (P, parental; G, gefitinib-exposed; E, erlotinib-exposed; G+N, gefitinib-exposed + 24 h NSC 74859; E+N, erlotinib-exposed + 24 h NSC 74859). Data are shown as the mean from three independent experiments."Early apoptotic cells, $\mathrm{P}<0.05$; " total cells positive for Annexin V, P $<0.05$. Statistical difference: One-way ANOVA/Dunnett's test, compared with the parental group. Representative graphs obtained by flow cytometry analysis after double staining with Annexin V-FITC and propidium iodide. (B) Effect of chemotherapy on poly(ADP-ribose) polymerase (PARP) cleavage. H1299 parental and TKI-exposed cells were incubated with four chemotherapeutic agents for $48 \mathrm{~h}(10 \mu \mathrm{M}$ DDP, $1 \mu \mathrm{M}$ paclitaxel, $40 \mu \mathrm{M}$ gemcitabine or $20 \mu \mathrm{M}$ pemetrexed), separation of cell proteins $(30 \mu \mathrm{g})$ by SDS/PAGE and reaction with antibodies against cleaved PARP. GAPDH was used as a loading control. (C) The cell cycle phases (G0/G1, G2/M and S phase) of both parental and TKI-exposed NSCLC cells were analyzed by flow cytometry using propidium iodide staining of DNA content.

downstream molecules: AKT, MAPK family (Erk, p38, JNK), STAT3, etc. (Fig. 3). Interestingly, we observed that phosphorylated AKT and STAT3 (at both Ser727 and Tyr705 sites) levels were substantially increased after exposure to TKI for 4 weeks, compared with parental H1299 cells. However, there was no significant increase in the level of the other phosphorylated molecules including Erk, p38, JNK and mTOR.

To investigate the relationship between AKT and STAT3 in the signal pathway, we used two PI3K inhibitors together (LY294002, $2 \mu \mathrm{M}$ and AS605240, $10 \mathrm{nM}$ ) and STAT3 inhibitor (NSC 74859, $20 \mu \mathrm{M}$ ) to treat H1299 parental cells with or without EGF $(50 \mathrm{ng} / \mathrm{ml})$. PI3K inhibition was associated with a significant reduction in P-AKT regardless of adding EGF or not, whereas level of P-STAT3 (Tyr705) showed obvious upregulation. Conversely, after incubation of STAT3 inhibitor, P-STAT3 (Tyr705) was considerably decreased, whereas P-AKT upregulated (Fig. 4A). Our data indicated that these two molecules were compensatory to each other in H1299 parental cells. To further investigate the relationship between these two proteins, along with exposure of TKI, we analyzed P-STAT3 and P-AKT expression in TKI-exposed cells. Our study revealed interesting data on these two molecules interaction (Fig. 4B). Similar to parental cells, PI3K inhibitors result in a downregulation of P-AKT and increase of P-STAT3, while NSC 74859 treatment resulted in downregulation of both P-STAT3 and P-AKT. These data suggest that EGFR-TKIs 


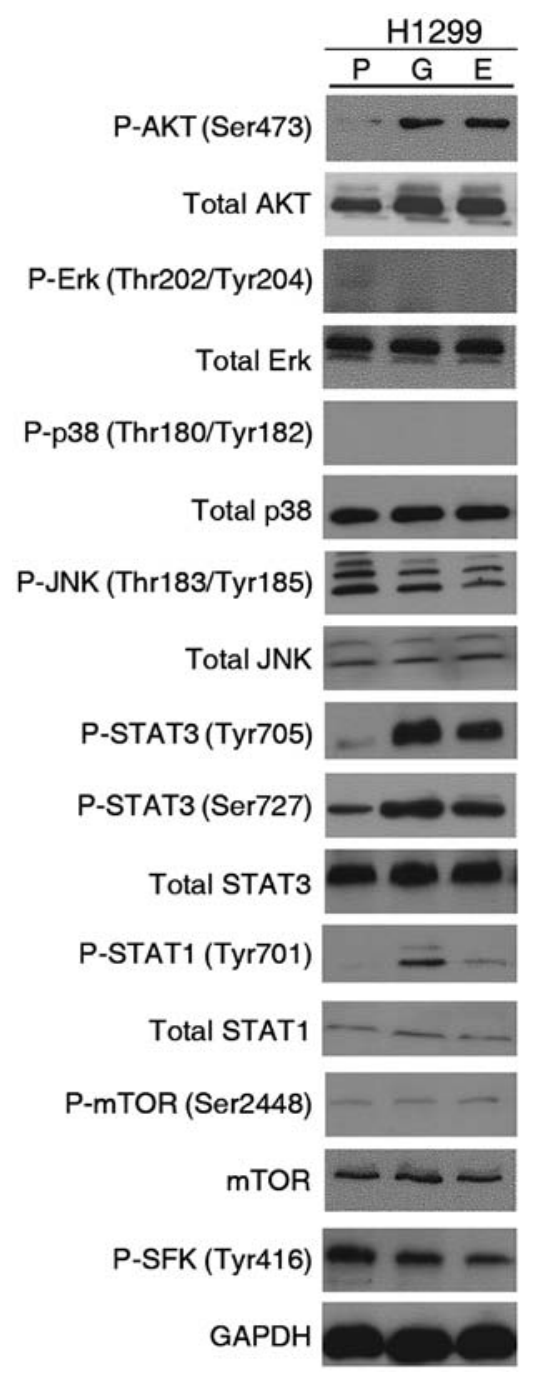

Figure 3. TKI exposure results in activation of signal transducer and activator of transcription 3 (STAT3). Western blot analyses for several important proteins of the EGFR signaling pathway and their phosphorylated forms using site-specific antibodies in both parental and TKI-exposed cells. GAPDH was used as a loading control. H1299 cells were treated with TKI (gefitinib, $10 \mu \mathrm{M}$; erlotinib, $5 \mu \mathrm{M}$ ) for 4 weeks. $\mathrm{P}$, parental; $\mathrm{G}$, gefitinib-exposed; E, erlotinib-exposed.

exposure results in role changes of STAT3 and AKT by which STAT3 becomes a regulator of the AKT signal. This also indicates that STAT3 plays a more important role in response to EGFR inhibition in EGFR-wt NSCLC cells.

To further assess the involvement of STAT3, we isolated nuclear and cytosolic fractions for immunoblotting assays. As shown in Fig. 5, a basal level of STAT3 was detectable in the nuclei of H1299 parental cells, as well as in the cytosol. Extracts from cells exposed to TKI for 4 weeks showed decreased cytosolic STAT3 and increased nuclear translocation.

To validate whether P-STAT3 increased in EGFR-wt NSCLC in vivo, we established a model using tumor xenografts with subcutaneously implanted H1299 cells. The tumor-bearing mice were gavaged once daily with gefitinib $(100 \mathrm{mg} / \mathrm{kg})$ or erlotinib $(100 \mathrm{mg} / \mathrm{kg})$ for 4 weeks. The levels of P-STAT3 (Tyr705) and P-STAT3 (Ser727) as well as total STAT3 in tumors were analyzed by western blot analysis (Fig. 6A) and immunohistochemistry (Fig. 6B). Consistent

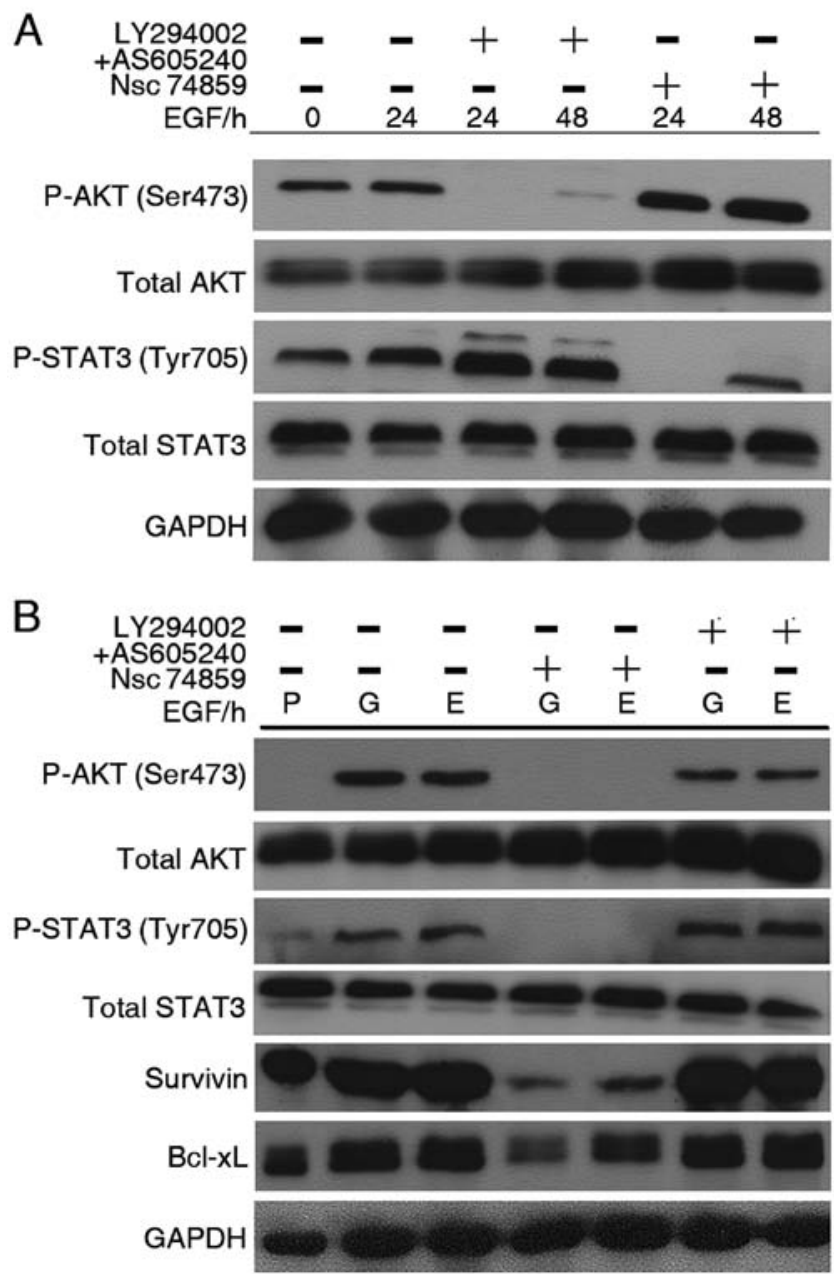

Figure 4. Chemical inhibitor of signal transducer and activator of transcription 3 (STAT3) suppress the activation of AKT in TKI-exposed non-small cell lung cancer (NSCLC) cells, but not in parental cells. (A) Time-course analysis of AKT and STAT3 molecule activation in EGF $(50 \mathrm{ng} / \mathrm{ml})$-treated H1299 parental cells. H1299 cells were treated with combination of two PI3K inhibitors (LY294002, $2 \mu \mathrm{M}$ and AS605240, $10 \mathrm{nM}$ ) or STAT3 inhibitor (NSC 74859, $20 \mu \mathrm{M}$ ). (B) H1299 TKI-exposed cells were treated with combination of two PI3K inhibitors (LY294002, $2 \mu \mathrm{M}$ and AS605240, $10 \mathrm{nM}$ ) or STAT3 inhibitor (NSC 74859, $20 \mu \mathrm{M}$ ) in the presence of gefitinib or erlotinib. After 48 h, cell lysed analyzed by western blot analysis. Equal loading and transfer were shown by repeat probing with GAPDH. P, parental; G, gefitinib-exposed; E, erlotinib-exposed.

with the results obtained in vitro, we observed increased levels of P-STAT3 (Tyr705) and P-STAT3 (Ser727) in xenografts exposed to EGFR-TKIs in comparison to the group gavaged daily with normal saline.

STAT3 activation results in chemoresistance by increasing anti-apoptotic signals, cell cycle arrest and epithelial-mesenchymal transition (EMT) in EGFR-wt NSCLC cells. To determine the role of STAT3 in chemoresistance caused by TKI exposure, we first examined the effect of STAT3 inhibition with the pharmacological inhibitor NSC 74859 on sensitivity to the four cytotoxic agents. STAT3 inhibition induced by $24 \mathrm{~h}$ incubation with $20 \mu \mathrm{M}$ NSC 74859 greatly recovered the cytotoxic effect of the different cytotoxic agents as indicated in Fig. 1A. We also found that caspase-3 activity induced by cytotoxic agents had a significant recovery after STAT3 inhibition by NSC 74859 (Fig. 1B). Moreover, flow 


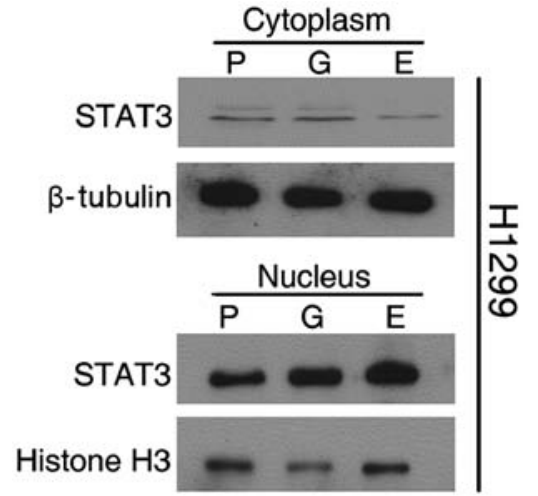

Figure 5. Signal transducer and activator of transcription 3 (STAT3) is a critical molecule in the development of chemoresistance in TKI-exposed non-small cell lung cancer (NSCLC) cells. Translocation of STAT3 from the cytoplasm to the nucleus occurs in TKI-exposed cells. Cytoplasmic and nuclear extracts were subjected to western blot analysis using an anti-STAT3 antibody. $\beta$-tubulin and Histone $\mathrm{H} 3$ were used as loading controls for cytoplasmic and nuclear protein extracts, respectively. The inhibitor of nuclear translocation, leptomycin B was present at $50 \mathrm{nM}$ for $6 \mathrm{~h}$ before extraction. $\mathrm{P}$, parental; G, gefitinib-exposed; E, erlotinib-exposed.

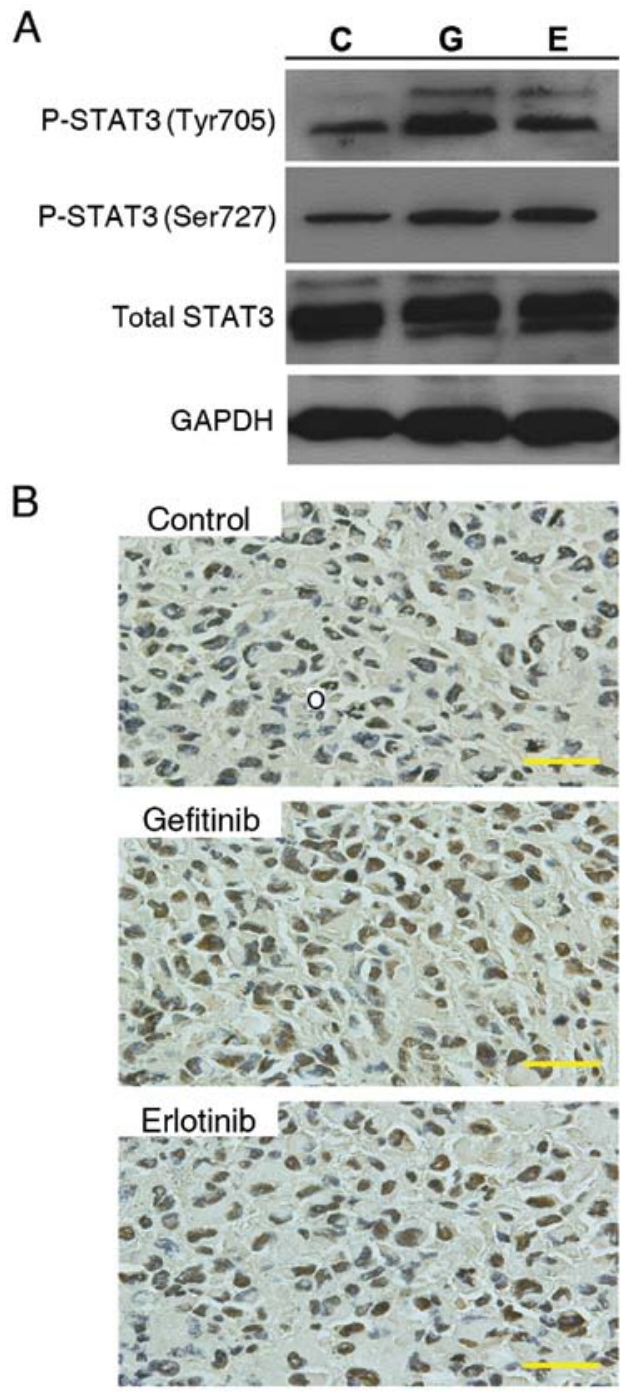

Figure 6. Epidermal growth factor receptor-tyrosine kinase inhibitors (EGFR-TKIs) increase the expression of P-STAT3 in H1299 tumor xenograft model. (A) Western blot analyses for P-STAT3 in tissue samples taken from the tumor xenograft. C, control group; G, gefitinib-exposed; E, erlotinib-exposed. (B) Immunohistochemical staining of P-STAT3 in tumor section (200x).

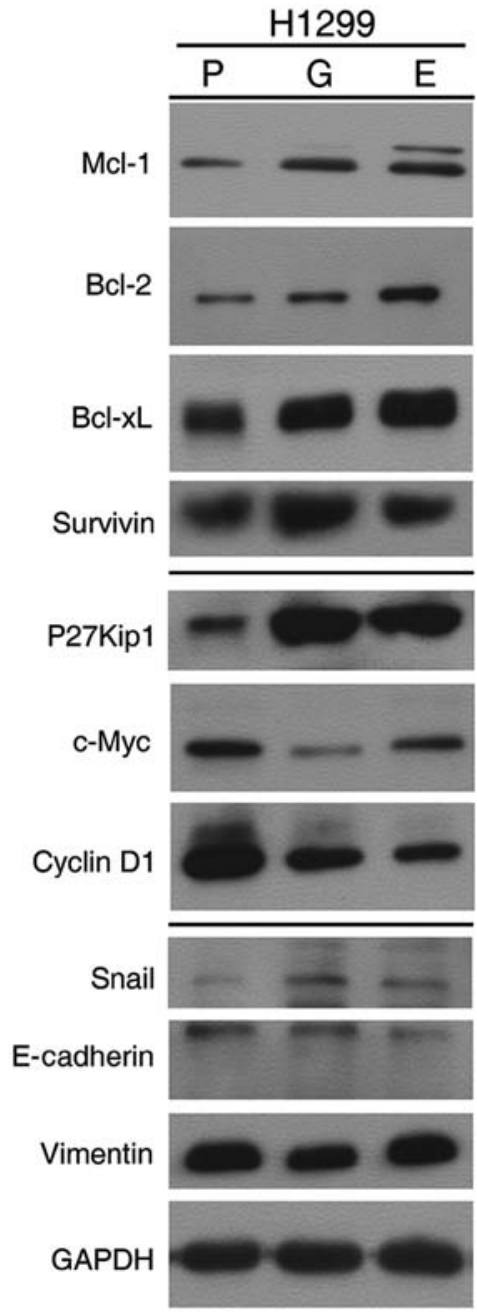

Figure 7. Analysis of signal transducer and activator of transcription 3 (STAT3) signal pathway proteins potentially involved in chemoresistance. Western blot analyses of proteins that are anti-apoptotic, induce cell cycle arrest, and induce epithelial-mesenchymal transition (EMT). GAPDH was used as a loading control. P, parental; G, gefitinib-exposed; E, erlotinibexposed.

cytometric analysis of Annexin V-stained cells demonstrated that STAT3 inhibition increased apoptosis induced by cytotoxic agents (Fig. 2A). Therefore, targeting STAT3 with a specific inhibitor actually reversed chemoresistance, and this indicates that STAT3 activation may play a vital role in altering the signal pathways operating after TKI exposure in EGFR-wt NSCLC cell lines.

To explore the potential mechanisms underlying STAT3 activation-induced drug resistance, we assessed the abundance of proteins of several STAT3-targeted genes (Fig. 7). The expression levels of four anti-apoptotic proteins (Mcl-1, Bcl-2, Bcl-xL and survivin) were greater in H1299 cells following prolonged TKI exposure. This suggests that activating STAT3 by prolonged TKI exposure impairs the ability of cytotoxic agents through the effects of these anti-apoptotic proteins. The levels of, P27Kip1, c-Myc and cyclin D1 also were measured; both cyclin D1 and c-Myc became less abundant, whereas increased level of P27KIP1 was detected in cells exposed to TKI. These findings may explain the G1-S phase arrest by TKIs (Fig. 2C). In addition, we also observed that P-STAT1 (Tyr701) levels in all three TKI-exposed cell 


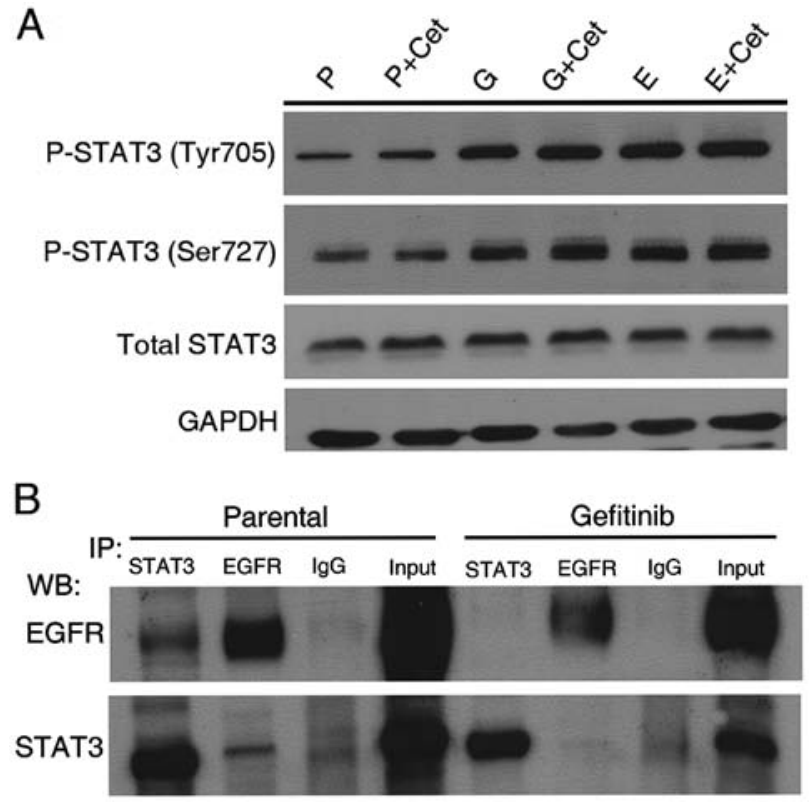

Figure 8. EGFR protein has no effect on activation of signal transducer and activator of transcription 3 (STAT3). (A) H1299 cells were incubated with TKI alone or in combination with $2.5 \mu \mathrm{g} / \mathrm{ml}$ cetuximab (Cet) for $48 \mathrm{~h}$, followed by immunoblotting analysis. P, parental; G, gefitinib-exposed; E, erlotinib-exposed. (B) Co-immunoprecipitation between STAT3 and EGFR in H1299 and H292 cells. Non-denatured extracts prepared from parental and gefitinib-exposed cells were immunoprecipitated with anti-STAT3, anti-EGFR or a control IgG antibody. The immune complexes and the input ( $20 \%$ of the extracts used in the immunoprecipitation step) were analyzed by immunoblotting with antibodies specific to STAT3 or EGFR.
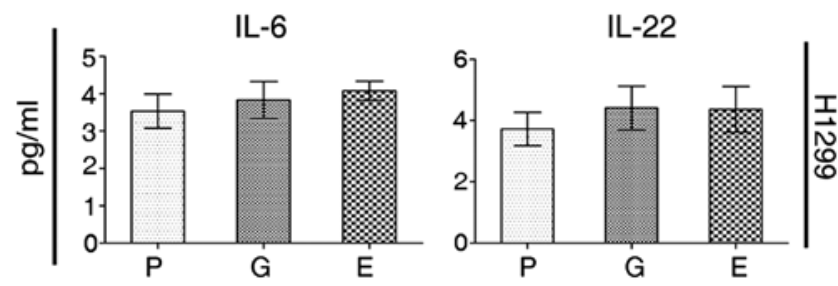

Figure 9. Quantification of IL-6 and -22 by ELISA in cell culture supernatant. IL-6 and -22 levels in the culture medium after being serum-starved and epidermal growth factor receptor-tyrosine kinase inhibitor (EGFR-TKI)-exposed for $48 \mathrm{~h}$ were measured using ELISA. Data are shown as mean $\pm \mathrm{SD}$; statistical difference: one-way ANOVA/Dunnett's test, compared with the parental group. Experiments were repeated three times. P, parental; G, gefitinib-exposed; E, erlotinib-exposed.

lines were markedly higher than parental cells. In this study, we observed that Snail, a key regulator of EMT, expression in TKI-exposed cells was slightly higher in the exposed compared to the parental cells. We also detected decreased levels of E-cadherin and increased levels of vimentin in TKI-exposed cells.

STAT3 activation does not depend on EGFR. To examine the possibility that STAT3 is activated through a direct physical interaction between STAT3 and EGFR, an immunoprecipitation assay was performed. As shown in Fig. 8B, parental H1299 cells exhibit slight binding between STAT3 and EGFR in the normal physiological state, however, after long-term exposure to EGFR-TKI the binding of STAT3 to EGFR was inhibited when identical amounts of total proteins were used

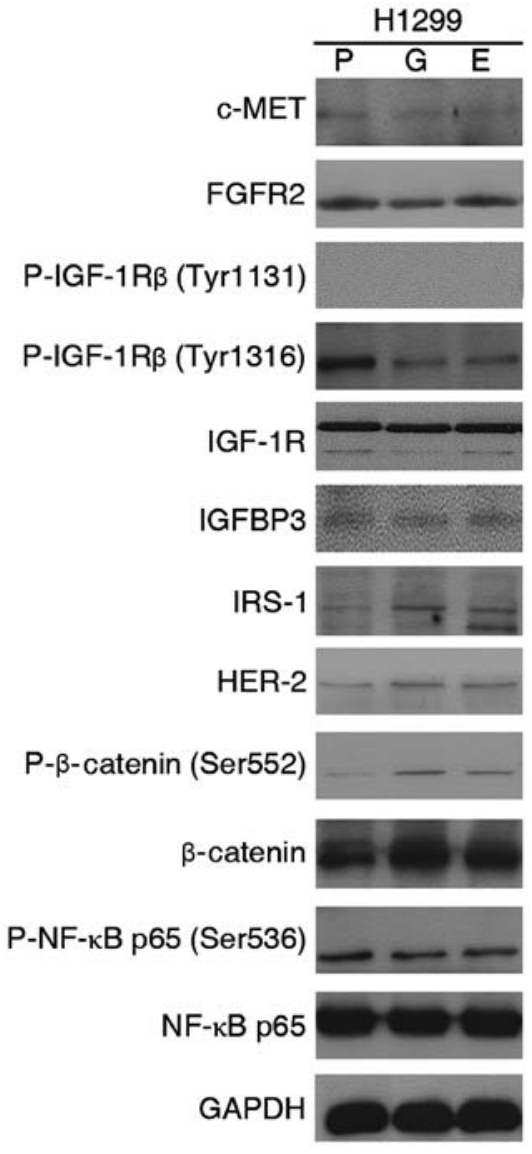

Figure 10. Detection of several potential mechanisms of chemoresistance induced by epidermal growth factor receptor-tyrosine kinase inhibitors (EGFR-TKIs). Western blot analyses of some proteins that are involved in resistance of EGFR-TKIs which may contribute to chemoresistance. GAPDH was used as a loading control. P, parental; G, gefitinib-exposed; E, erlotinib-exposed.

for pulldown by an anti-STAT3 or -EGFR antibody. To further explore whether the mechanism of STAT3 activation was independent of EGFR, we used cetuximab as a treatment to block EGFR dimerization in EGFR-TKI-exposed cells. As shown in Fig. 8A, cetuximab did not affect the abundance of P-STAT3.

STAT3 activation is independent of IL- 6 and -22. In order to explain the mechanisms of activation of STAT3, we measured the level of IL-6 and -22 in the supernatant of culture media harvested from our cell experiments. For H1299 cells there was no significant difference between the levels of these cytokines released from TKI-exposed and parental cells (Fig. 9).

$I G F-1 R$ and c-MET are not involved in chemoresistance. We examined whether there were other mechanisms, which had been reported to potentially contributed to chemoresistance, including several major proteins of IGF-1R signaling, c-MET, phosphorylated NF-KB p65 and so on (Fig. 10). However, our studies revealed there were no significant difference between parental and TKI-exposed cells.

Targeting STAT3 augments the efficacy of cytotoxic drugs against cells possessing EGFR with both L858R and T790M mutations. Given that chemotherapy is a primary treatment 
A

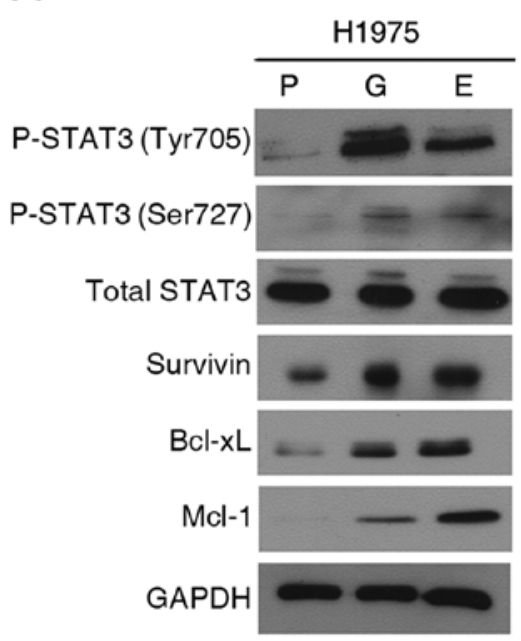

B

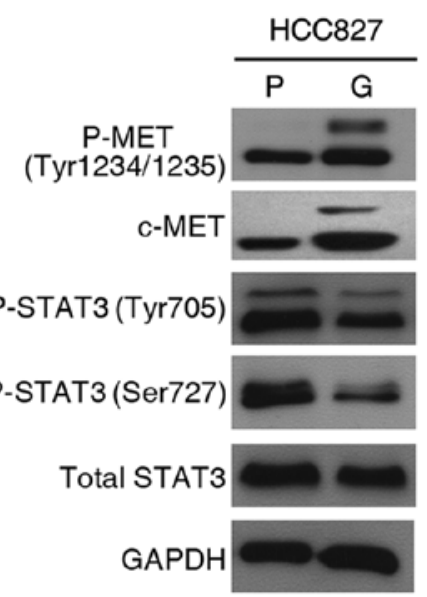

C

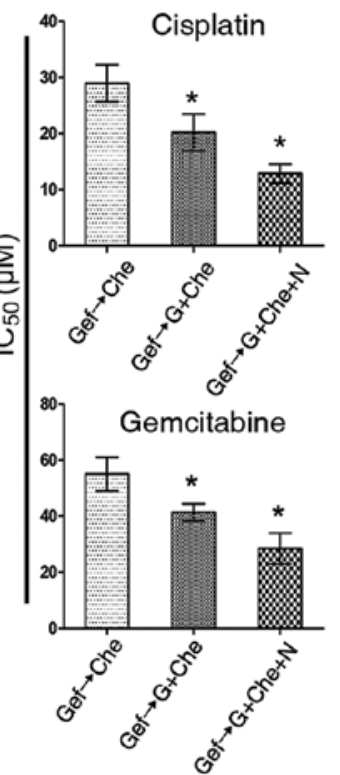

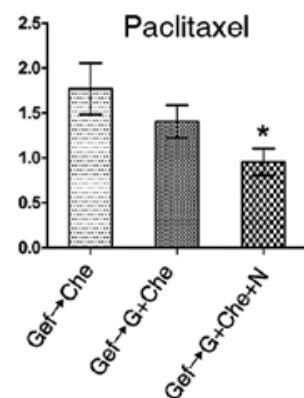

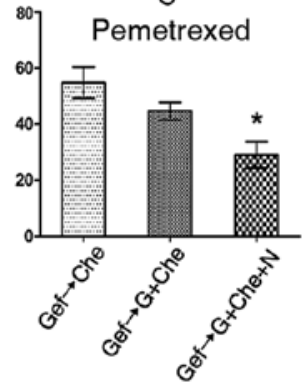

D
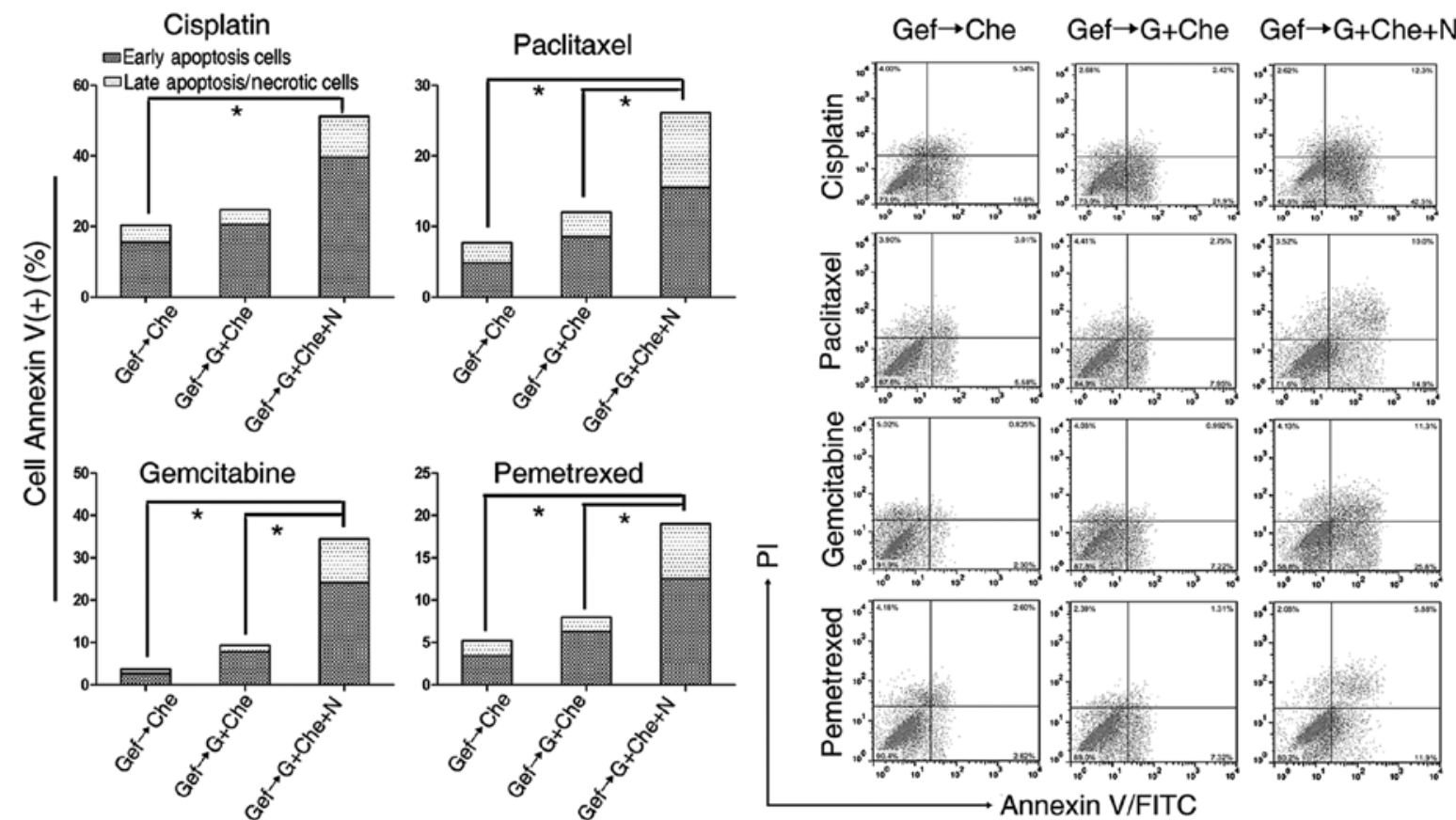

Figure 11. Signal transducer and activator of transcription 3 (STAT3) inhibition enhances the antitumor effect of combining gefitinib with cytotoxic drugs in non-small cell lung cancer (NSCLC) cells bearing the EGFR T790M mutation. (A and B) STAT3 activation was observed in H1975 cells exposed to TKI, but not in HCC827 cells long-term exposed to gefitinib. P, parental; G, gefitinib-exposed; E, erlotinib-exposed. (C) Determination of IC ${ }_{50}$ using the CCK-8 cell number assay in H1975 cells long-term exposed to gefitinib. Gef $\rightarrow$ Che, gefitinib removed and replaced by chemotherapeutic agents; Gef $\rightarrow$ Gef + Che, gefitinib continued and supplemented with chemotherapeutic agents; Gef $\rightarrow \mathrm{Gef}+\mathrm{Che}+\mathrm{N}$, gefitinib continued and supplemented with chemotherapeutic agents and NSC 74859. (D) Analysis of H1975 cells by flow cytometry to assess apoptosis. Groups as in (C). Concentrations of chemotherapeutic agents used: cisplatin (DDP) $10 \mu \mathrm{M}$, paclitaxel $1 \mu \mathrm{M}$, gemcitabine $20 \mu \mathrm{M}$ or pemetrexed $20 \mu \mathrm{M}$. Data shown as mean $\pm \mathrm{SD}$; ${ }^{*} \mathrm{P}<0.05$; statistical difference: one-way ANOVA/Dunnett's test, compared with the control group. Experiments were repeated three times.

choice following EGFR-TKI treatment failure, we investigated whether EGFR-mt NSCLC cells with resistance to EGFR-TKI generate chemoresistance by similar mechanisms. H1975 cells (harboring two mutations of EGFR) were treated with gefitinib for 4 weeks and HCC827 cells (in which resistance to EGFR-TKI is due to c-MET amplification) were treated with gefitinib for 6 months to simulate clinical acquired TKI resistance. When, we assessed STAT3 after gefitinib treatment, we found that its phosphorylation was increased in $\mathrm{H} 1975$ cells (a representative of acquired EGFR-TKI resistance with the EGFR T790M mutation) but not in HCC827 cells (in which resistance to EGFR-TKI via c-MET amplification) (Fig. 11A and B). Subsequently, H1975 cells, gefitinib-exposed for 4 weeks were treated in three groups: i) chemotherapeutic drug alone; ii) chemotherapeutics drugs in combination with gefitinib; and iii) NSC 74859 and cytotoxic drugs with gefitinib together (Fig. 11C). Compared 
with cytotoxic drugs alone, all cells treated with gefitinib and cytotoxic drugs concurrently exhibited increased cytotoxicity. The $\mathrm{IC}_{50}$ values were decreased, suggesting a synergistic or addictive interaction between gefitinib and the heterogeneous group of the four cytotoxic drugs (DDP, paclitaxel, gemcitabine and pemetrexed). The addition of NSC 74859 to the combination of gefitinib and cytotoxic drugs resulted in distinctly enhanced cytotoxic effects (Fig. 11D). These data suggest that failure of EGFR-TKI treatment may also result in activation of STAT3, and thus targeting the STAT3 pathway maybe helpful for subsequent chemotherapy.

\section{Discussion}

Increased expression of EGFR has been found in $40-80 \%$ of NSCLC cases (14-16). Therefore, multiple approaches have been developed in order to inhibit EGFR, such as competition for the extracellular domain by monoclonal antibodies (cetuximab) or the inhibition of EGFR tyrosine kinase activity by small molecules interacting with the intracellular domain (erlotinib, gefitinib and afatinib).

The characterization of EGFR-mts was a crucial discovery associated with high efficacy of biomarker-driven treatment (17). As a result, EGFR-TKIs are now the treatment of choice for patients with EGFR-mutated tumors $(18,19)$.

For chemotherapy-naive advanced NSCLC patients, several clinical trials with biomarker-driven selection (EURTAC, OPTIMAL, WJTOG3405, and NEJ002) have proven that a statistically significant and clinically relevant increase in PF was obtained using TKIs compared to chemotherapy $(4,20-22)$. Nevertheless, subgroup analysis based on molecular analyses (IPASS and First-SIGNAL) revealed that chemotherapy was significantly better than EGFR-TKIs in EGFR-wt patients $(3,8)$. It has been proposed that some EGFR-wt or status-unknown NSCLC patients who undergo first-line EGFR-TKI treatment have a worse prognosis and lower response rate to chemotherapy, according to the results of Gridelli et al in the TORCH study (Tarceva or chemotherapy) (10).

Despite the fact that EGFR-TKIs are not generally more efficacious than chemotherapy for unselected patients, and is not recommended to treat patients whose EGFR status is unknown, in practice it is reasonable for gefitinib or erlotinib to be used as an exploratory treatment for patients whom have never smoked or have been light smokers. For instance, EGFR-mts occur in $~ 50 \%$ of Asian patients with NSCLC (23). Standard EGFR mutation analysis requires a minimum amount of tumor tissue, however, for a large proportion of advanced NSCLC patients this may not be available. In addition, methods such as 'liquid biopsy' that study circulating lung cancer cells or that analyze 'free tumor DNA' in the plasma still have a lot of problems to conquer, including a low concordance rate between plasma and in situ biopsy (24). Usually, after undergoing a 4-week exploratory treatment, the tumor response will be reassessed using response evaluation criteria in solid tumors (RECIST) compared with base-line data. Following a tumor response of partial response (PR) or stable disease (SD), TKI treatment will continue, otherwise, TKI will be replaced with chemotherapy.

Due to the likelihood that chemotherapy-naive patients with EGFR-wt could possibly be treated with EGFR-TKIs, we decided to evaluate the influence of EGFR-TKIs on subsequent chemotherapy in a culture system model. The outcomes showed that EGFR-TKIs had an adverse effect on the subsequent chemotherapy for any of the four agents we tested: DDP, paclitaxel, gemcitabine and pemetrexed. Our findings strongly support that continuous exposure to EGFR-TKIs before chemotherapy results in chemoresistance in EGFR-wt NSCLC cells.

Our study found that continuous EGFR-TKI exposure actually induces high-level activation of STAT3 signaling and rescue of AKT/mTOR. Interestingly, the inhibition of STAT3 completely deleted the phosphorylation of AKT, but not vice versa. Moreover, inhibition of PI3K did not affect the level of phospho-AKT. These results showed that sustaining EGFR-TKI exposure deprived function of PI3K, an upstream regulator of AKT, while overactivation of STAT3 replaced the role of PI3K to re-foster the AKT/mTOR pathway. Our data support previous findings that STAT3 activation regulates AKT activation upstream of AKT pathway in EGFR-wt NSCLC when exposed to EGFR-TKI (25), and indicates that overactivation of STAT3 plays a critical role in response to long-term EGFR-TKI exposure.

During consecutive, long-term exposure to EGFR-TKIs, STAT3 is activated, as shown by increased levels of P-STAT3, DNA binding, and transcriptional activity $(26,27)$. The finding in our present study is that the activation of STAT3 is tightly correlated with the signals for survival and growth arrest. H1299 cells responded with an upregulation of Bcl-2, Bcl-xL, Mcl-1 and survivin which represent anti-apoptotic signals. Additionally, downregulation of c-Myc, cyclin D1 and an increase of P27KIP1 indicated cell growth arrest. These findings contradict recent reports of a direct correlation among cyclin D1, c-Myc and STAT3 (28), but the downregulation of c-Myc by activation of STAT3 in tumor tissues has also been reported by other researchers (29), and cyclin D1 potentially creates a negative feedback loop onto STAT3 (30). In addition, STAT1 has been demonstrated to suppress c-Myc and cyclin D1 expression as a negative transcriptional regulator which relates to cell cycle arrest and an increase of P-STAT1 was observed in TKI-exposed cells in our experiments (31-33). Previous investigations have reported that STAT3 could lead to EMT, which may be helpful for chemoresistance (34-36), EMT was observed in TKI-exposed cells. Therefore, our study showed that STAT3 activation in response to continuous EGFR-TKI exposure further resulted in chemoresistance via multiple mechanisms.

To support the hypothesis that STAT3 was the major effector molecule, we used an inhibitor of STAT3 (NSC 74859) to treat cells long-term exposed to TKIs, and examined the sensitivity of these cells to cytotoxic agents in vitro. We found that the TKI preconditioned cells regained sensitivity to cytotoxic agents, to a large degree. Considering our results obtained both in vitro and in vivo, we believe that we can provide a plausible explanation for these discordant results; that STAT3 play a major role in the adverse effects.

It is well known that IGF-1R and NF- $\mathrm{BB}$ signaling, as well as MET amplification involving in EGFR-TKI resistance both de novo and acquired $(37,38)$. As we have shown here that STAT3 has negative effects on cytotoxic 
agents, IGF-1R and MET were not essential partners for this in $\mathrm{H} 1299$ cells.

Activation of STAT3 has been reported to occur through binding of the IL- 6 family of cytokines to the gp130 receptor (35). High levels of IL-6, which was secreted by EGFR-TKI, was induced in several cell lines $(39,40)$. Inconsistent with the known effects of IL-6 on STAT3 signaling (40-42), we found IL-6 as well as IL-22 was not essential for activating STAT3 in long-term EGFR-TKI-exposed NSCLC. Our study also showed a reduction in the level of EGFR/STAT3 complex in continuously TKI-exposed cells, differently from in short-exposed (25). As cetuximab has no effect on the activation of STAT3, we incline to believe that a negative correlation exists between activation of STAT3 and EGFR in this study. It will be important to further examine how STAT3 be activated in our follow-up studies.

The EGFR TKI-resistant cell line H1975 harbors a double mutation (L858R and T790M) in the EGFR gene. T790M is sometimes present as a minor allele before EGFR-TKI therapy and accounts for about half of the acquired resistance cases.

Several clinical trials have suggested that second-line erlotinib treatment was effective in those who had prior disease control with first-line gefitinib. Other research shows that continuation of an EGFR-TKI with chemotherapy compared to chemotherapy alone significantly increases the ORR but not PFS and OS in patients with advanced NSCLC and acquired TKI resistance (43). Indeed, in our in vitro results, EGFR TKI with chemotherapy was more effective than chemotherapy alone against H1975 TKI-exposed cells. Considering that the activation of the STAT3 signaling pathway has also been demonstrated both in H1975 TKI-exposed and parental cells, we combined NSC 74859 with gefitinib and chemotherapy agents in H1975 TKI-exposed cells. There was a significant synergistic killing effect from combination treatment with NSC 74859, which is in accordance with results from several other researchers.

In our study, we focused on EGFR TKIs as frontline agents prior to chemotherapy. Our results raise the possibility that exposure to EGFR-TKIs possibly activates STAT3. Similarly, Haura et al (44) found that patients with early-stage NSCLC who received 4 weeks of treatment with gefitinib $(250 \mathrm{mg}$ daily) before surgical resection had abundant expression of P-STAT3 in their surgically resected tumors. Thus, the use of EGFR-TKI as exploratory treatment on patients with unknown EGFR-mt status must be considered with caution and prudence.

In conclusion, whether there is de novo or acquired resistance to chemotherapy by persistent activation of STAT3, a combination strategy of chemotherapeutic with STAT3 inhibitor may be beneficial for NSCLC patients. We believe that our in vitro and in vivo xenograft models sufficiently support that targeting STAT3 is a strategy worth considering for circumventing EGFR-TKI resistance in patients.

\section{Acknowledgements}

This study was supported by the National Major Project of China (2011ZX09302-001-01) and the National Natural Science Foundation of China (Beijing, China) (81472197).

\section{References}

1. Riely GJ, Politi KA, Miller VA and Pao W: Update on epidermal growth factor receptor mutations in non-small cell lung cancer Clin Cancer Res 12: 7232-7241, 2006.

2. Ono M and Kuwano M: Molecular mechanisms of epidermal growth factor receptor (EGFR) activation and response to gefitinib and other EGFR-targeting drugs. Clin Cancer Res 12: 7242-7251, 2006.

3. Mok TS, Wu YL, Thongprasert S, et al: Gefitinib or carboplatin-paclitaxel in pulmonary adenocarcinoma. N Engl J Med 361: 947-957, 2009.

4. Zhou C, Wu YL, Chen G, et al: Erlotinib versus chemotherapy as first-line treatment for patients with advanced EGFR mutation-positive non-small-cell lung cancer (OPTIMAL, CTONG-0802): a multicentre, open-label, randomised, phase 3 study. Lancet Oncol 12: 735-742, 2011.

5. Maemondo M, Inoue A, Kobayashi K, et al: Gefitinib or chemotherapy for non-small-cell lung cancer with mutated EGFR. N Engl J Med 362: 2380-2388, 2010.

6. Laurie SA and Goss GD: Role of epidermal growth factor receptor inhibitors in epidermal growth factor receptor wild-type non-small-cell lung cancer. J Clin Oncol 31: 1061-1069, 2013.

7. Gatzemeier U, Pluzanska A, Szczesna A, et al: Results of a phase III trial of erlotinib (OSI-774) combined with cisplatin and gemcitabine (GC) chemotherapy in advanced non-small cell lung cancer (NSCLC). J Clin Oncol ASCO 22: abs. 7010, 2004.

8. Han JY, Park K, Kim SW, et al: First-SIGNAL: first-line single-agent Iressa versus gemcitabine and cisplatin trial in never-smokers with adenocarcinoma of the lung. J Clin Oncol 30: 1122-1128, 2012.

9. Pirker R, Pereira JR, von Pawel J, et al: EGFR expression as a predictor of survival for first-line chemotherapy plus cetuximab in patients with advanced non-small-cell lung cancer: analysis of data from the phase 3 FLEX study. Lancet Oncol 13: 33-42, 2012.

10. Gridelli C, Ciardiello F, Gallo C, et al: First-line erlotinib followed by second-line cisplatin-gemcitabine chemotherapy in advanced non-small-cell lung cancer: the TORCH randomized trial. J Clin Oncol 30: 3002-3011, 2012.

11. Wu JY, Shih JY, Yang CH, et al: Second-line treatments after first-line gefitinib therapy in advanced nonsmall cell lung cancer. Int J Cancer 126: 247-255, 2010.

12. Kumar A, Petri ET, Halmos B and Boggon TJ: Structure and clinical relevance of the epidermal growth factor receptor in human cancer. J Clin Oncol 26: 1742-1751, 2008.

13. Kolch W and Pitt A: Functional proteomics to dissect tyrosine kinase signalling pathways in cancer. Nat Rev Cancer 10: 618-629, 2010.

14. Sebastian S, Settleman J, Reshkin SJ, Azzariti A, Bellizzi A and Paradiso A: The complexity of targeting EGFR signalling in cancer: from expression to turnover. Biochim Biophys Acta 1766: 120-139, 2006.

15. Normanno N, De Luca A, Bianco C, et al: Epidermal growth factor receptor (EGFR) signaling in cancer. Gene 366: 2-16, 2006.

16. Irmer D, Funk JO and Blaukat A: EGFR kinase domain mutations - functional impact and relevance for lung cancer therapy. Oncogene 26: 5693-5701, 2007.

17. Kobayashi S, Boggon TJ, Dayaram T, et al: EGFR mutation and resistance of non-small-cell lung cancer to gefitinib. N Engl J Med 352: 786-792, 2005.

18. Garassino MC, Martelli O, Broggini M, et al: Erlotinib versus docetaxel as second-line treatment of patients with advanced non-small-cell lung cancer and wild-type EGFR tumours (TAILOR): a randomised controlled trial. Lancet Oncol 14: 981-988, 2013.

19. Yang J, Cheng Y, Zhao M, Zhou K, Yan H and Zhang L: A phase II trial comparing pemetrexed with gefitinib as the second-line treatment of nonsquamous NSCLC patients with wild-type EGFR (CTONG0806). J Clin Oncol ASCO 31: abs. 8042, 2013.

20. Rosell R, Carcereny E, Gervais R, et al: Erlotinib versus standard chemotherapy as first-line treatment for European patients with advanced EGFR mutation-positive non-small-cell lung cancer (EURTAC): a multicentre, open-label, randomised phase 3 trial. Lancet Oncol 13: 239-246, 2012.

21. Mitsudomi T, Morita S, Yatabe Y, et al: Gefitinib versus cisplatin plus docetaxel in patients with non-small-cell lung cancer harbouring mutations of the epidermal growth factor receptor (WJTOG3405): an open label, randomised phase 3 trial. Lancet Oncol 11: 121-128, 2010. 
22. Kobayashi K, Inoue A, Maemondo M, et al: First-line gefitinib versus first-line chemotherapy by carboplatin (CBDCA) plus paclitaxel (TXL) in non-small cell lung cancer (NSCLC) patients (pts) with EGFR mutations: a phase III study (002) by North East Japan Gefitinib Study Group. J Clin Oncol ASCO 27: abs. 8016, 2009.

23. Sequist LV, Bell DW, Lynch TJ and Haber DA: Molecular predictors of response to epidermal growth factor receptor antagonists in non-small-cell lung cancer. J Clin Oncol 25: 587-595, 2007.

24. Maheswaran S, Sequist LV, Nagrath S, et al: Detection of mutations in EGFR in circulating lung-cancer cells. N Engl J Med 359: 366-377, 2008

25. Wu K, Chang Q, Lu Y, et al: Gefitinib resistance resulted from STAT3-mediated Akt activation in lung cancer cells. Oncotarget 4: 2430-2438, 2013.

26. Barré B, Vigneron A, Perkins N, Roninson IB, Gamelin E and Coqueret O: The STAT3 oncogene as a predictive marker of drug resistance. Trends Mol Med 13: 4-11, 2007.

27. Dauer DJ, Ferraro B, Song L, et al: Stat 3 regulates genes common to both wound healing and cancer. Oncogene 24: 3397-3408, 2005.

28. Ai T, Wang Z, Zhang M, et al: Expression and prognostic relevance of STAT3 and cyclin D1 in non-small cell lung cancer. Int J Biol Markers 27: e132-e138, 2012.

29. Yamanaka Y, Nakajima K, Fukada T, Hibi M and Hirano T: Differentiation and growth arrest signals are generated through the cytoplasmic region of gp130 that is essential for Stat3 activation. EMBO J 15: 1557-1565, 1996.

30. Germain D and Frank DA: Targeting the cytoplasmic and nuclear functions of signal transducers and activators of transcription 3 for cancer therapy. Clin Cancer Res 13: 5665-5669, 2007.

31. Dimberg A, Karlberg I, Nilsson K and Oberg F: Ser727/Tyr701phosphorylated Stat1 is required for the regulation of c-Myc, cyclins, and p27Kip1 associated with ATRA-induced G0/G1 arrest of U-937 cells. Blood 102: 254-261, 2003.

32. Ramana CV, Chatterjee-Kishore M, Nguyen H and Stark GR: Complex roles of Stat 1 in regulating gene expression. Oncogene 19: 2619-2627, 2000.

33. Dimco G, Knight RA, Latchman DS and Stephanou A: STAT1 interacts directly with cyclin D1/Cdk4 and mediates cell cycle arrest. Cell cycle 9: 4638-4649, 2010.
34. Yamashita S, Miyagi C, Fukada T, Kagara N, Che YS and Hirano T: Zinc transporter LIVI controls epithelial-mesenchymal transition in zebrafish gastrula organizer. Nature 429: 298-302, 2004.

35. Yadav A, Kumar B, Datta J, Teknos TN and Kumar P: IL-6 promotes head and neck tumor metastasis by inducing epithelial-mesenchymal transition via the JAK-STAT3-SNAIL signaling pathway. Mol Cancer Res 9: 1658-1667, 2011.

36. Wang $\mathrm{H}$, Zhang $\mathrm{G}$, Zhang $\mathrm{H}$, et al: Acquisition of epithelial-mesenchymal transition phenotype and cancer stem cell-like properties in cisplatin-resistant lung cancer cells through AKT/ $\beta$-catenin/Snail signaling pathway. Eur J Pharmacol 723: 156-166, 2014.

37. Lin L and Bivona TG: Mechanisms of resistance to epidermal growth factor receptor inhibitors and novel therapeutic strategies to overcome resistance in NSCLC patients. Chemother Res Pract 2012: 817297, 2012.

38. Chen Y-F and Fu L-W: Mechanisms of acquired resistance to tyrosine kinase inhibitors. Acta Pharm Sin B 1: 197-207, 2011.

39. Yeh HH, Lai WW, Chen HH, Liu HS and Su WC: Autocrine IL-6-induced Stat3 activation contributes to the pathogenesis of lung adenocarcinoma and malignant pleural effusion. Oncogene 25: 4300-4309, 2006.

40. Lee HJ, Zhuang G, Cao Y, Du P, Kim HJ and Settleman J: Drug resistance via feedback activation of Stat 3 in oncogene-addicted cancer cells. Cancer Cell 26: 207-221, 2014

41. Kim SM, Kwon OJ, Hong YK, et al: Activation of IL-6R/JAK1/STAT3 signaling induces de novo resistance to irreversible EGFR inhibitors in non-small cell lung cancer with T790M resistance mutation. Mol Cancer Ther 11: 2254-2264, 2012.

42. Song L, Rawal B, Nemeth JA and Haura EB: JAK1 activates STAT3 activity in non-small-cell lung cancer cells and IL-6 neutralizing antibodies can suppress JAK1-STAT3 signaling. Mol Cancer Ther 10: 481-494, 2011.

43. Goldberg SB, Oxnard GR, Digumarthy S, et al: Chemotherapy with Erlotinib or chemotherapy alone in advanced non-small cell lung cancer with acquired resistance to EGFR tyrosine kinase inhibitors. Oncologist 18: 1214-1220, 2013.

44. Haura EB, Sommers E, Song L, Chiappori A and Becker A: A pilot study of preoperative gefitinib for early-stage lung cancer to assess intratumor drug concentration and pathways mediating primary resistance. J Thorac Oncol 5: 1806-1814, 2010. 